\title{
Expression of Transcriptional Factors of GATA4, GATA6 and SF-1 in the Developmental Process of Rat Adrenal Cortex; the Possible Contribution of GATA4 in Limited Expression before Zonal Establishment
}

\author{
Yuichiro Tomita ${ }^{1}$, Shinobu Umemura ${ }^{2}$, Tamaki Sasou ${ }^{3}$, Hideaki Hasegawa ${ }^{3}$, \\ Fumiko Mitani $^{4}$, Osamu Shinohara ${ }^{1}$, Iekuni Ichikawa ${ }^{1}$ and R. Yoshiyuki Osamura ${ }^{2}$ \\ ${ }^{1}$ Department of Pediatrics, ${ }^{2}$ Department of Pathology and ${ }^{3}$ Department of Laboratories for Structure and Function Research, \\ Tokai University School of Medicine, Isehara and ${ }^{4}$ Department of Biochemistry, Keio University School of Medicine, Tokyo
}

Received October 9, 2002; accepted November 8, 2002

\begin{abstract}
The adrenal cortex consists of three zones producing different hormones: zona glomerulosa $(z G)$, zona fasciculata $(\mathrm{zF})$ and zona reticularis (zR). Accumulated knowledge has revealed the contribution of transcriptional factors to the functional development of the adrenal cortex, however, the regulatory mechanisms have not yet been fully understood. We studied the expression and localization of transcriptional factors, SF1, GATA4 and GATA6 by immunohistochemistry in the developmental process, establishing three zones of the rat adrenal cortex. The development of the adrenal cortex was monitored by the expression of cortical enzymes, P45011 $\beta$, $\mathrm{P} 450$ aldo, and P450scc on gestational days $15,16,18,19,20$ (G15, G16, G18, G19, G20) and postnatal days 1, 2, 20 $(\mathrm{P} 1, \mathrm{P} 2, \mathrm{P} 20)$ in Spraque-Dawley rats. GATA4 expression was observed in a
\end{abstract}

limited number of cortical cells from early gestation (G15) to the neonatal period (P20) before complete establishment of the cortical zones. Nearing the final phase of ZG establishment (P20), GATA4 changed its intracellular localization from the nuclei to the perinuclear region. Another noteworthy finding is that GATA6 translocated from the cytoplasm into the nuclei on $\mathbf{G 1 9}$, followed by a leaning distribution of SF-1 in ZG (P1) and changing the distribution of SF-1 from $Z G$ to $Z F$ parallel to the establishment of ZG (P20). In conclusion, we report that GATA4 was detected in the immature phase of the adrenal cortex in limited expression but diminished in the mature adrenal cortex. GATA4 may participate in the gene transcription required for the development of the adrenal cortex.

Key words: Adrenal cortex, Transcriptional factor, GATAs, SF-1, Functional zonation

\section{Introduction}

The adrenal gland consists of two functionally distinct endocrine glands, the medulla and cortex, within a single encapsulated organ. The cortex derives from mesodermal cells attached to the coelomic cavity lining adjacent to the urogenital ridge. The migration of primitive cells begins around the 15th day of fetal life and continues until term in rats. The cortex is further subdivided into three zones, zona glomerulosa $(\mathrm{zG})$, zona fasciculata $(\mathrm{zF})$ and zona reticu-

Correspondence to: Yuichiro Tomita, MD, Department of Pediatrics, Tokai University School of Medicine, Bohseidai, Isehara 259-1193, Kanagawa, Japan. laris (zR). Different hormones are produced in each zone: the mineralocorticoid from $\mathrm{zG}$, glucocorticoid from $\mathrm{zF}$, and so-calledadrenal androgens from $z R[17,18]$. Our understanding of the development of tissue specific cells is advancing with accumulating knowledge of the regulatory mechanisms of gene expression and roles of transcriptional factors. A transcriptional factor, steroidogenic factor-1 (SF-1), is known to contribute to the development of gonadal organs and adrenal gland [13]. No specific ligand for SF-1 is known to increase the functional activity, therefore SF-1 is considered to be an orphan receptor. An SF-1 knock out mouse model showed that SF-1 is critical for sexual development and the organogenesis of the adrenal gland. SF-1 regulates many gene expressions of key proteins on the 
pituitary-gonadal axis, for example, Mullerian-inhibiting substance (MIS), glycoprotein, $\alpha$-subunit, LH, GnRH, steroidgenic acute regulatory protein (StAR), DAX-1, PRL receptor and ACTH receptor $[19,24]$. The gene expression regulated by SF-1 cooperates with DAX-1 and/or WT-1 [5].

Another lineage of knowledge for transcriptional factors is being accumulated for the GATA family, which consists of six GATA proteins binding to a consensus DNA sequence of WGATAR [26]. GATA proteins are expressed in a broad spectrum of tissues, including the brain, gut, heart, hematopoietic cells, pituitary and gonads $[21,22,27]$, and can be separated into two subgroups, the hematopoietic group (GATA1/2/3) and the cardiac group (GATA4/5/6) [26]. Recently, GATA4 and GATA6 were demonstrated to play a role in the gonads $[7,8,26]$. GATA is expressed from the early phase of development of both the ovary and testis. GATA4 and GATA6 are also detected in mouse ovarian follicles. In the case of the adrenal gland, a lack of GATA4 expression in adult rat made led us to assume that GATA4 does not participate in adrenal gland development. To the best of our knowledge, the roles of GATA in adrenal gland development have not been well understood. To gain an insight into the roles of GATAs in adrenal gland development and functions, we examined the expression of GATAs in relation to SF-1 and zonal formation the using adrenal gland of fetal and post natal rats.

\section{Materials and Methods}

\section{Animals}

Sprague-Dawley rats were obtained from Clea Japan. Inc. (Japan). They were housed in a conventional room at a controlled temperature of $23 \pm 1{ }^{\circ} \mathrm{C}$ and a relative humidity of $55 \pm 5 \%$, in a $12 \mathrm{hr}$ light-dark cycle. The rats were fed a standard commercial laboratory diet (CRF-1, Oriental Yeast
Co., Tokyo, Japan) and tap water ad libitum according to the guidelines of Tokai University Animal Care for animal experimentation. We examined the adrenal glands of three rats in each stage of the developmental process as follows. The rats were anesthetized with diethyl ether and killed by exsanguination. The examined adrenal glands were of fetus rats on the 15 (G15), 16 (G16), 19 (G19) and 20 (G20) days of gestation and of neonatal rats on the 1 (P1) and 20 (P20) days after birth. Ten-weeks-old rats were studied as adults. The adrenal gland of the rats, except for G15 and G16, were removed and subjected to the following process, embedding in O.C.T. compound (Sakura Finetek U.S.A., Inc., Torrance, CA) and frozen in dry-ice hexan. The whole bodies of the fetuses of G15 and G16 were embedded in O.C.T. compound and frozen.

\section{Immunohistochemistry}

Steroidogenic enzymes and transcriptional factors were immunohistochemically examined using frozen sections. The adrenal glands, except for G15, were serially cut in $6 \mu \mathrm{m}$-thickness and mounted on 3-amino propyltrimethoxy silane-coated slides (Matsunami Glass, Osaka, Japan). The whole mounted bodies from G15 were serially cut and mounted, and the adrenal glands were stained with H\&Estain every third section. Six to eight unstained sections of each adrenal gland were available for the antibodies examined. Commercially available antibodies for transcriptional factors, GATA4, GATA6 (Santa Cruz Biotechnology, Inc., Santa Cruz, CA) and SF-1 (Affinity Bioreagents, Inc., CO, U.S.A) were used. The antibodies against the steroidogenic enzymes of the cytochrome P450 side chain cleavage enzyme were purchased from Chemicon International, Inc. (Temecula, CA, U.S.A). The polyclonal antibodies of cytochrome P450 11 $\beta$-hydroxylase (P45011 $\beta$ ) and cytochrome $\mathrm{P} 450$ aldosterone synthase (P450aldo) were originated by a

Table 1. Protocols for immunohistochemical stainings

\begin{tabular}{|c|c|c|c|c|c|c|c|c|c|c|}
\hline & GATA4 & GATA6 & NG & SF-1 & NR & $\mathrm{p} 450 \mathrm{scc}$ & NR & $\mathrm{p} 45011 \mathrm{~b}$ & p450aldo & NR \\
\hline Fixation & \multicolumn{3}{|c|}{$4 \%$ PFA 2 min } & \multicolumn{2}{|c|}{$\begin{array}{l}\text { Acetone } 10 \mathrm{~min} \\
\rightarrow 4 \% \text { PFA } 2 \mathrm{~min}\end{array}$} & \multicolumn{2}{|c|}{$4 \%$ PFA 5 min } & \multicolumn{3}{|c|}{$\begin{array}{l}\text { Acetone+Chloroform } 5 \mathrm{~min} \\
\rightarrow \text { Acetone } 1 \mathrm{~min} \\
\rightarrow \text { Acetone } 1 \mathrm{~min}\end{array}$} \\
\hline \multirow{2}{*}{ Reduction of non-specific reaction } & \multicolumn{3}{|c|}{$\begin{array}{l}\mathrm{H}_{2} \mathrm{O}_{2}-\mathrm{MeOH} \\
15 \mathrm{~min}\end{array}$} & & & \multicolumn{2}{|c|}{$\begin{array}{l}\mathrm{H}_{2} \mathrm{O}_{2}-\mathrm{MeOH} \\
15 \mathrm{~min}\end{array}$} & & & \\
\hline & \multicolumn{3}{|c|}{$\begin{array}{l}5 \% \text { normal rabbit serum } \\
15 \text { min }\end{array}$} & \multicolumn{7}{|c|}{$\begin{array}{l}5 \% \text { normal sheep serum } \\
15 \mathrm{~min}\end{array}$} \\
\hline Incubation with primary antibodies & \multicolumn{10}{|c|}{ at $4^{\circ} \mathrm{C}$ for over night } \\
\hline Reduction of non-specific reaction & \multicolumn{3}{|c|}{$\begin{array}{l}5 \% \text { normal rabbit serum } \\
15 \text { min }\end{array}$} & \multicolumn{7}{|c|}{$\begin{array}{l}5 \% \text { normal sheep serum } \\
15 \mathrm{~min}\end{array}$} \\
\hline \multirow{2}{*}{ Incubation with secondary antibodies } & \multicolumn{5}{|c|}{ biotin-conjugated anti-goat IgG } & \multirow{3}{*}{\multicolumn{5}{|c|}{$\begin{array}{l}\text { HRP-conjugated anti rabbit IgG } \\
120 \mathrm{~min}\end{array}$}} \\
\hline & & $30 \mathrm{~min}$ & & 60 & & & & & & \\
\hline \multirow{2}{*}{ Detection } & \multicolumn{5}{|c|}{$\begin{array}{l}\text { streptoavidin-biotinylated complex } \\
30 \mathrm{~min}\end{array}$} & & & & & \\
\hline & \multicolumn{10}{|c|}{ DAB 60 min } \\
\hline
\end{tabular}

NG, negative control for antibodies produced by goat; NR, negative control for antibodies produced by rabbit. 
member of authors (FM) [11]. The immunohistochemical procedures for each antibody are summarized in the Table 1. Briefly, fixation for GATAs and P450scc was performed in $4 \%$ paraformaldehyde (PFA) in $10 \mathrm{mM}$ phosphate buffer (PB), $\mathrm{pH} \mathrm{7.0,} \mathrm{at} \mathrm{room} \mathrm{temperature} \mathrm{(RT)} \mathrm{for} 2 \mathrm{~min}$ and $5 \mathrm{~min}$, and for SF-1 100\% acetone at RT for $10 \mathrm{~min}$ followed by $4 \%$ PFA for $2 \mathrm{~min}$. The sections were fixed in a mixture of acetone and chloroform at RT for 5 min for P450aldo and $\mathrm{P} 45011 \beta$ as described previously [11]. To reduce nonspecific reactions, the sections were incubated with $5 \%$ normal serum in phosphate buffered saline (PBS) and hydrogen peroxide-methanol solution for GATAs and P450scc, and with $5 \%$ normal serum for other antibodies. All of the primary antibodies were incubated at $4^{\circ} \mathrm{C}$ overnight. The sections were rinsed in $0.01 \mathrm{M} \mathrm{PBS}, \mathrm{pH} 7.4$ for $15 \mathrm{~min}$, and incubated with secondary antibodies. For GATAs, the sections were incubated with biotin-conjugated anti-goat IgG at RT for 30 min followed by streptoavidin-biotin complex (the VECTASTAIN Elite ABC Kit (Vector Laboratories, Inc.). Because the adrenal gland is a biotin-rich tissue, we had to exclude non-specific reaction for endogenous biotin. It is well known that non-specific reaction for endogenous biotin is reduced by $4 \%$ PFA fixation, which was confirmed by negative reaction using non-immunized serum. The other antibodies were stained by an indirect method by incubation with HRP-conjugated anti rabbit IgG at RT for 60-120 min. The positive controls were the heart for GATAs and the adrenal gland for other antibodies. Non-specific reaction was excluded by negative control using non-immunized serum for all of the antibodies used in the present study.

\section{Results}

\section{Functional zonation of the adrenal cortex; Expression of p450scc, p45011 $\beta$, and p450aldo (Fig. 1)}

The adrenal gland could be detected as a nodular structure located above the kidney with a vague encapsulation surrounded by a spindle mesenchymal cell layer merging with the connective tissue. The adrenal gland on G19 was detectable as a distinct organ with a thin fibrous capsule. ZG became distinguishable around G16 as a layer with aggregated cortical cells containing round nuclei. The adrenal medulla was well developed from G20. The structure of the adrenal gland on P20 was almost fully developed, compatible with those of adult rats.

P450scc was expressed in the cytoplasm of cortical cells throughout the three zones from G15, although the expression was very faint. On G19, p450scc was expressed strongly at the inner part (zF, zR) of the gland, and weakly in $\mathrm{zG}$. Distinction between the adrenal cortex and medulla was clearly documented on P20, which was highlighted by the P450scc expression in zR. P450scc expressing cells were increased in $\mathrm{zG}$ as the adrenal gland fully developed. In the adrenal gland of adult rats, scattered $\mathrm{P} 450 \mathrm{scc}$ positive cells were identified in the adrenal medulla. The cytoplasmic expression of $\mathrm{P} 450 \mathrm{scc}$ was consistent throughout the development process. The expression of P45011 $\beta$ was very faint but detectable on G16 at the inner part of the gland. Distribution in the adrenal cortex was consistently identified in $\mathrm{zF}$ and $\mathrm{zR}$ throughout the development with a rather stronger expression in $\mathrm{zF}$. Subcellular localization was in the cytoplasm. The P450aldo expression documented well the establishment of $\mathrm{zG}$. P450aldo was recognizable from $\mathrm{G} 20$ in a scattered manner. P450aldo positive cells were limited to the most outer part of the gland as a thin layer of zG, which was still irregular in thickness on P1. On $\mathrm{P} 20, \mathrm{zG}$ was completed as a regularly thickened layer, which is compatible with those of adult rats from the aspect of morphology (Fig. 1).

\section{Expression of transcriptional factors, GATA6, GATA4 and $S F-1$}

As shown in Fig. 2, the intracytoplasmic faint reaction for GATA6 on G15 was equivocal. GATA6 expression was clearly detected from G19 in the nuclei. The expression became more distinct as the rat matured to an adult. In contrast to the limited and focal expression of GATA4, the distribution of positive cells was diffusely identified in the adrenal cortex throughout the process.

A few, but distinctively positive, cells for GATA4 were detected on G15. A limited number of positive cells for GATA4 were also detected on G19 through P20 with a transient decrease on G20. In the adrenal gland of adult rats, GATA4 was undetectable. The expression pattern of GATA4 was peculiar and distinct from that of GATA6. Only a limited number of nuclear positive cells were detectable scattered in the cortex without a regular or organized distribution pattern. One noteworthy finding is that a positive reaction was detected in the perinuclear region in the $\mathrm{zF}$ of P1 and P20 (Fig. 3).

SF-1 is expressed in a more complex manner and is also recognizable from G15. The subcellular localization was consistently the intranuclear region throughout the developing process to an adult except on G20. Distribution in the adrenal gland was diffusely identified on G19. The positive cell population in the nuclei decreased transiently on G20, but an intracytoplastic positive reaction was diffusely identified. A higher percentage of positive cells was observed in the zona glomerulosa on P1 and in the zona fasciculata on $\mathrm{P} 20$. In the adrenal gland of adult rats, SF1 was expressed diffusely throughout the three zones of the cortical cells.

These findings were almost consistent among the bilateral adrenal glands of three rats, and the schematic is summarized in Fig. 4.

\section{Discussion}

In this study, GATA4 expression was observed in a small number of cortical cells during the gestational and early postnatal phase of the rat adrenal cortex, and it diminished after zonal establishment in the adult. The expression of SF-1 and GATA6 was observed more generally and consistently. Detailed observation of the zonal and intracellular localization showed several pieces of events, which 

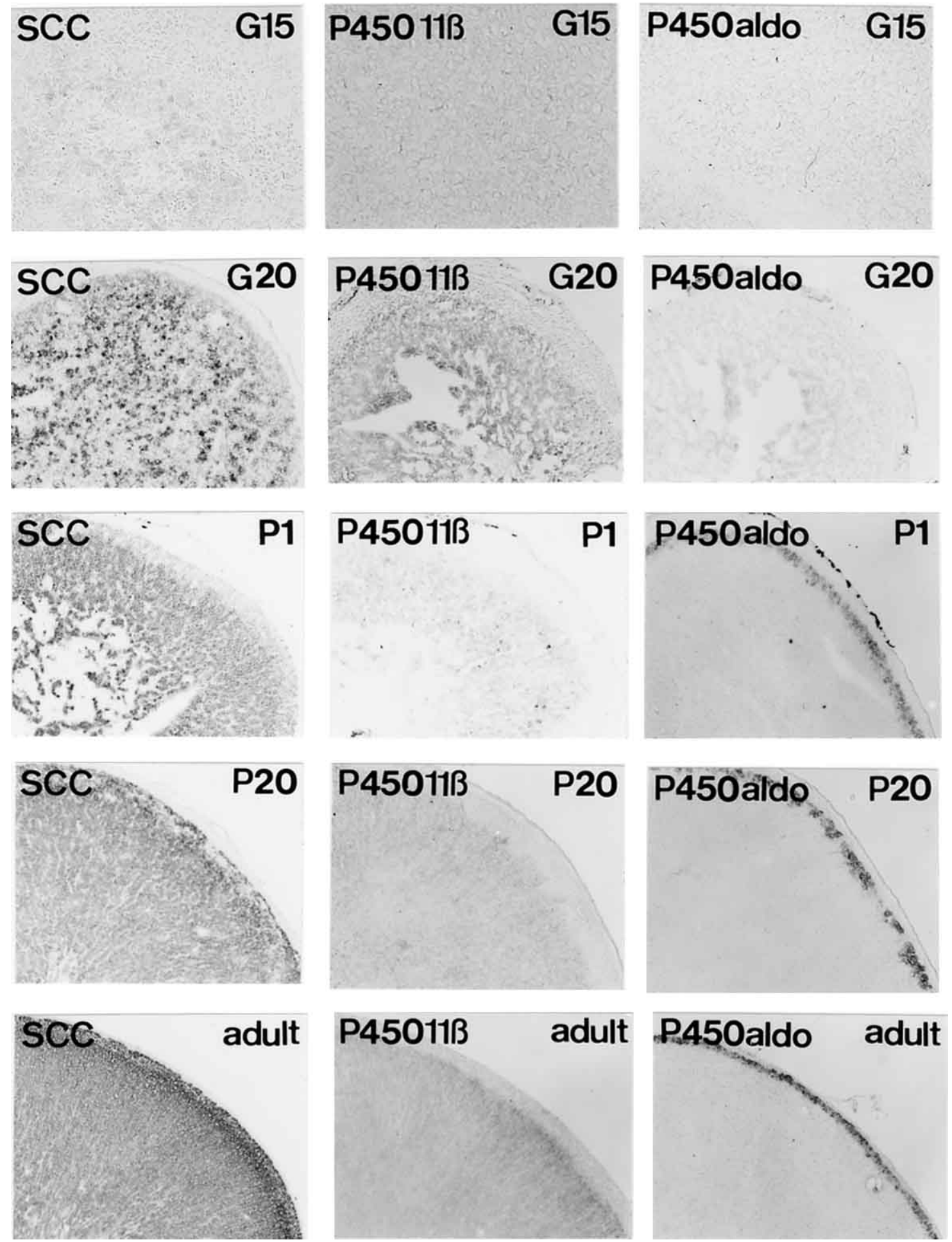

Fig. 1. Expression of $\mathrm{P} 450 \mathrm{scc}, \mathrm{P} 45011 \beta$ and $\mathrm{P} 450$ aldo in the rat adrenal cortex during the developmental process. P450scc is faintly detectable from G15. Immunostaining is distinctively identified in the cytoplasm of cortical cells from G19 throughout the process to an adult. Expression of P45011 $\beta$ on G15 is too faint to be demonstrated photographically. ZG lacks P45011 $\beta$ expression. P450aldo is recognized from G19, although the expression is clearly demonstrated from P1. $\times 75(\mathrm{G} 15), \times 60(\mathrm{G} 20), \times 50(\mathrm{P} 1, \mathrm{P} 20), \times 30$ (adult).

were suspected to occur serially in the process of zonal establishment. The nuclear localization of SF-1 (G15) proceeded to the translocation of GATA6 from the cytoplasm to nuclei (G19). After translocation, the leaning distribution of SF-1 was observed in zG (P1) followed by the changing distribution to $\mathrm{zF}$ and $\mathrm{zG}$ accompanied by the establishment 

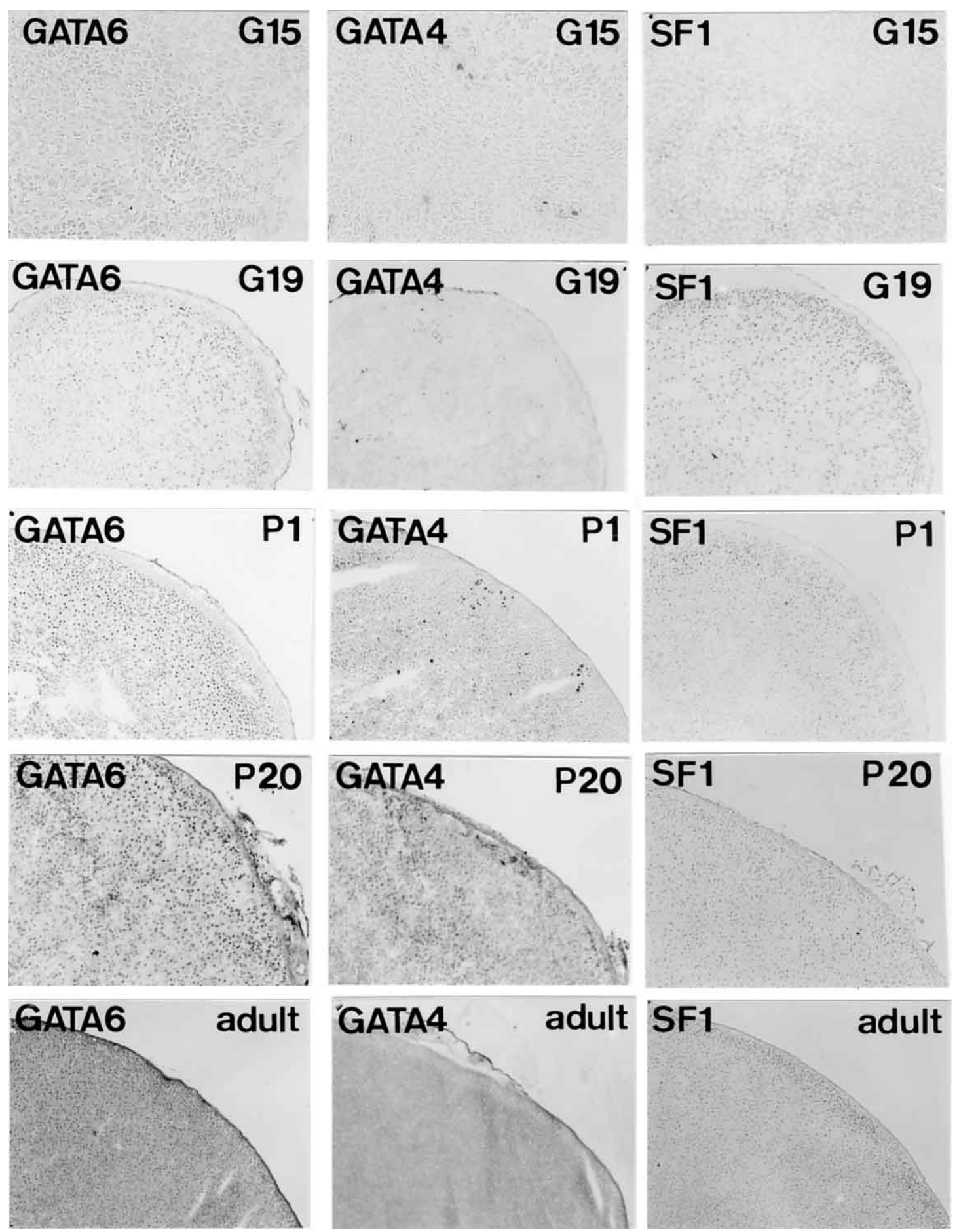

Fig. 2. Expression of transcriptional factors (GATA6, GATA4 and SF1) in the rat adrenal cortex during the developmental process. GATA6 expression is very faint on G15. Intranuclear positive reaction of GATA6 is demonstrated throughout the process in three layers. A few positive cells for GATA4 are detectable on G15, considerable numbers of positive cells are observed on G19, P1 and P20, but no expression is identified in adult rats. The positive cells are not diffuse, but have limited distribution. SF-1 positive cells are detectable on G15 throughout the process. Distribution of SF-1 positive cells leans to the zG on G19 and P1, shifting to the zR on P20, then distributes throughout the layers. $\times 75$ (G15), $\times 60(\mathrm{G} 19$ and $\mathrm{G} 20), \times 50(\mathrm{P} 1$ and P20), $\times 30$ (adult).

of $\mathrm{zG}$ on $\mathrm{P} 20$.

It has not been recognized that GATA4 has a biological role in the adrenal gland, because no expression of GATA4 has been shown in the adult adrenal gland and only a limited number of studies have been performed to examine GATAs in the adrenal gland $[6,8]$. Kiiveri et al. showed that no 

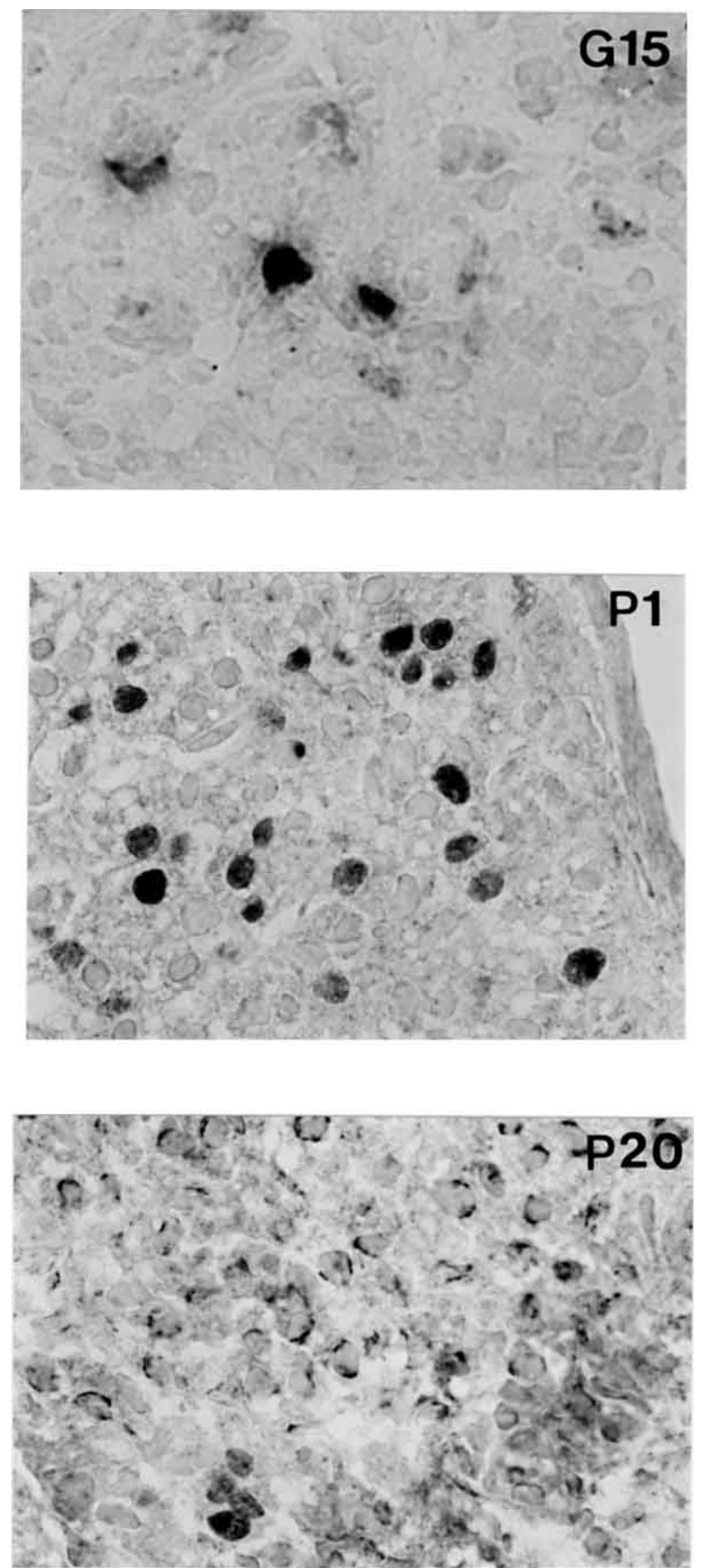

Fig. 3. Intracytoplasmic localization of GATA4. On G15 and P1, GATA4 is detected in the nuclei of the adrenocortical cells. The number of GATA4 positive cells increase on P20, but intracytoplasmic localization changes to the perinuclear space and cytoplasm. $\times 600$.

expression of GATA4 was detected in the mouse adrenal cortex of the adult and G18, in contrast to GATA6 [8]. Interestingly, in cortical tumors of mice and humans, GATA4 was diffusely expressed in tumor cells, but GATA6 was not. Our results of the adult adrenal gland was consistent with these reported results, and it was not surprising that the adrenal gland on G18 did not express GATA4 considering the limited expression of GATA4. GATA4 and
GATA6 are thought to have overlapping but distinct functions from studies of the hypothalamic-pituitary-gonadal axis. A study of the ovary revealed that GATA4 was expressed in potentially mitotic cells and was lost as cells terminally differentiated or became apoptotic [6]. Only a few positive nuclear cells detected in this study seem to reflect its inconstant role in unknown urgent needs. Perinuclear localization in $\mathrm{zF}$ reflects the inactive state of GATA4 protein that is ready to act or to be metabolized. Thus, the results of this study suggest the contribution of GATA4 to adrenal gland development as well as gonadal organs.

The adrenal gland on G15 was detected as an ovoid mass of aggregated glandular cells surrounded by mesenchymal cells. Encapsulation was not yet completed, but the glandular cells were plump and enlarged, and the cytologic features were distinct from surrounding cells. At that time, SF-1 and P450scc were diffusely expressed proceeding to the expression of GATAs. It is unlikely, therefore, that GATAs expression is essential for this initial phase of SF-1 and P450scc expression, although SF-1 has GATA-responsive elements in the promotor region. SF-1 has significant roles in the embryogenesis of steroidogenic organs. In the promoter region of many steroidogenic genes of $\mathrm{P} 450 \mathrm{scc}$ $[20,25], \mathrm{P} 45011 \beta$ [12], P450aldo [2], P45017 $\alpha$ [1, 28], $3 \beta$ HSD [9] and StAR [3, 23], and nonsteroidogeniec promoters like MIS [15], SF-1 sites have been detected [5, 19]. StAR is believed to support the transfer of hydrophobic cholesterol, a substrate of $\mathrm{P} 450 \mathrm{scc}$ for the production of pregnenolone, across the mitochondrial membrane. The simultaneous expression of SF-1 and P450scc can be explained by at least two mechanisms. One is the stimulous transcriptional activation of the p450scc gene through SF-1 responsive elements, and the other is via the transcriptional activation of the StAR gene by SF-1. Turning the eye to the human adrenal gland, expression of GATAs has not been documented, but SF-1 and P450scc were also demonstrated in developing adrenal gland [16]. In human adrenal glands of fetus, SF-1 was detected in the nuclei of adrenocortical parenchymal cells in almost all of the cells of the definitive, transitional and fetal zones throughout gestation. P450scc was detected in the cytoplasm of adrenocortical parenchymal cells in the transitional and fetal zones throughout gestation. These findings are similar to those of rat adrenal gland examined in the present study.

The state of SF-1 observed in this study provides us with interesting clues for discussion of regulatory mechanisms. First, the serial expression of GATA6 and accumulating SF-1 positive cells in $\mathrm{zG}$ may suggest an interaction between GATA6 and SF-1. Although it has not yet been fully understood, SF-1 has a GATA binding site in the promotor region, and the possible contribution of GATA to SF-1 transcription has been proposed [26]. Second, the shifting distribution of SF-1 from $\mathrm{zG}$ to $\mathrm{zF}$ on P20 suggests a mechanism for SF-1 expression as occasion demands. The enzymatic activity of $11 \beta$-hydroxylase is regulated by four genes (CYP11B1-4). CYP11B1 codes a $11 \beta$-hydroxylase $(\mathrm{p} 45011 \beta)$ in $\mathrm{zF} / \mathrm{zR}$, and a second gene (CYP11B2) 


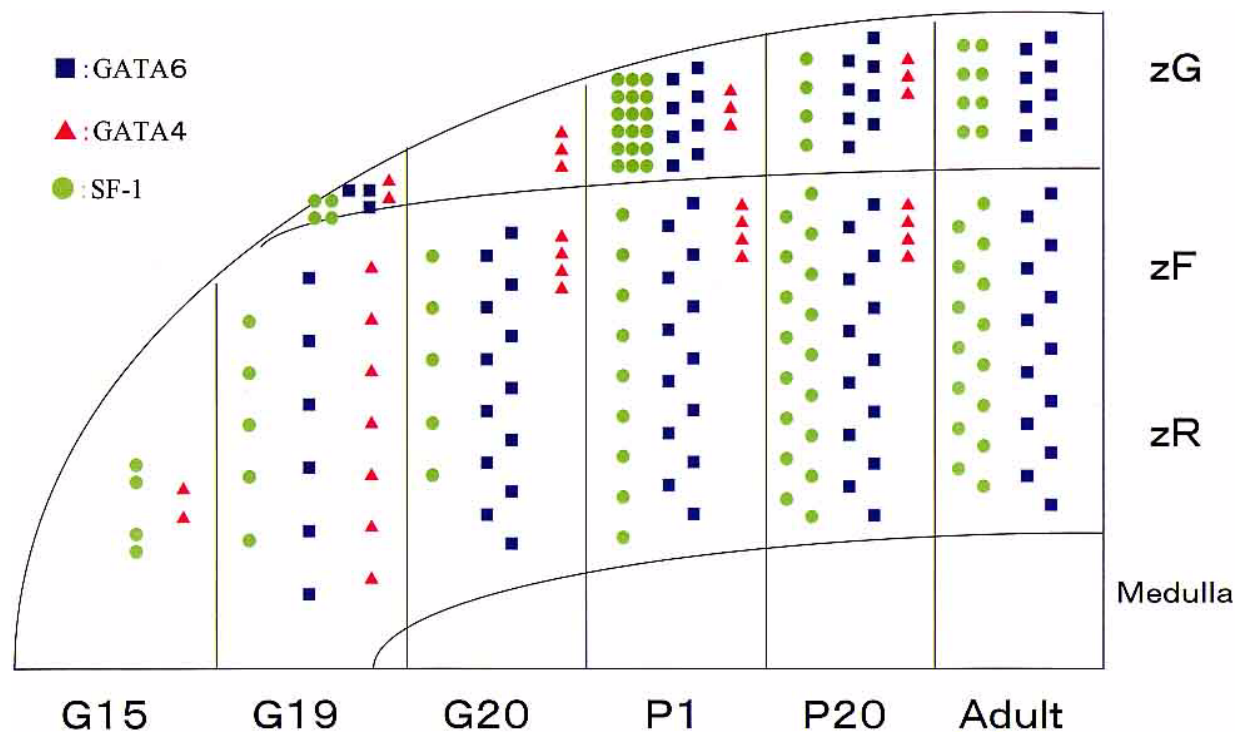

Fig. 4. Schematic summary of immunohistochemical results for transcriptional factors in adrenal gland development. The scheme indicates the relative number of positive cells distributed in each layer (zG, zona glomerulosa; zF, zona fasciculata; zR, zona reticularis). Transcriptional factors are shown in different colors (GATA6; blue, GATA4; pink, SF-1; green).

encodes an aldosterone synthase (p450aldo) in zG having three enzymatic activities, $11 \beta$-hydroxylase, 18-hydroxylase and 18-oxidase. CYP11B3 encodes a similar mRNA and protein to p45011 [10], and CYP11B4 is a pseudogene. It has been reported that CYP11B1 (bovine) [12] and CYP11B2 (mouse) [2] have SF-1-responsive sites. Thus they could be good models to demonstrate how SF-1 regulates different genes, which are to be expressed. The third issue of concern is the hormonal environment of the whole body. Little is known about the hormonal regulation of SF-1. It was reported that ACTH stimulation did not influence the transcription of SF-1 in a study using culture cells [4], although p45011 $\beta$ was stimulated by administration of ACTH with increased GSH-PO [14]. GnRH treatment, however, was reported to increase the SF-1 mRNA level in rat pituitaries [4]. It is easy to understand that there could be a changing internal environment from late gestation to the early the postnatal phase. The fluid composition and circulation system change drastically before and after birth, demanding increased mineralcorticoid and glucocorticoid for adaptation. The expression of SF-1 could be, thus, reciprocally regulated with the internal hormonal environment.

In conclusion, GATA4 was detected in the adrenal cortex from early gestational (G15) to the postnatal phase (P20) in limited expression. GATA4 may participate in the gene transcription required in the immature stage of cortical development.

\section{Acknowledgments}

We thank J. Itoh (Department of Laboratories for structure and Function Research, Tokai University) for providing excellent quality photography. We are also grateful to Dr. Y. Ikeya for advising how to obtain fresh-frozen sections at
G15.

\section{References}

1) Bakke, M. and Lund, J. (1995) Mutually exclusive interactions of two nuclear orphan receptors determine activity of a cyclic adenosine 3',5'-monophosphate-responsive sequence in the bovine CYP17 gene. Mol. Endocrinol. 9; 327-339.

2) Bogerd, A. M., Franklin, A., Rice, D. A., Schimmer, B. P. and Parker, K. L. (1990) Identification and characterization of two upstream elements that regulate adrenocortical expression of steroid 11 beta-hydroxylase. Mol. Endocrinol. 4; 845-850.

3) Caron, K. M., Ikeda, Y., Soo, S. C., Stocco, D. M., Parker, K. L. and Clark, B. J. (1997) Characterization of the promoter region of the mouse gene encoding the steroidogenic acute regulatory protein. Mol. Endocrinol. 11; 138-147.

4) Haisenleder, D. J., Yasin, M., Dalkin, A. C., Gilrain, J. and Marshall, J. C. (1996) GnRH regulates steroidogenic factor-1 (SF-1) gene expression in the rat pituitary. Endocrinology 137; 5719-5722.

5) Hanley, N. A., Rainey, W. E., Wilson, D. I., Ball, S. G. and Parker, K. L. (2001) Expression profiles of SF-1, DAX1, and CYP17 in the human fetal adrenal gland: potential interactions in gene regulation. Mol. Endocrinol. 15; 57-68.

6) Heikinheimo, M., Ermolaeva, M., Bielinska, M., Rahman, N. A., Narita, N., Huhtaniemi, I. T., Tapanainen, J. S. and Wilson, D. B. (1997) Expression and hormonal regulation of transcription factors GATA-4 and GATA- 6 in the mouse ovary. Endocrinology $138 ; 3505-3514$.

7) Ketola, I., Rahman, N., Toppari, J., Bielinska, M., Porter-Tinge, S. B., Tapanainen, J. S., Huhtaniemi, I. T., Wilson, D. B. and Heikinheimo, M. (1999) Expression and regulation of transcription factors GATA-4 and GATA- 6 in developing mouse testis. Endocrinology 140; 1470-1480.

8) Kiiveri, S., Siltanen, S., Rahman, N., Bielinska, M., Lehto, V. P., Huhtaniemi, I. T., Muglia, L. J., Wilson, D. B. and Heikinheimo, M. (1999) Reciprocal changes in the expression of transcription factors GATA-4 and GATA-6 accompany adrenocortical tumorigenesis in mice and humans. Mol. Med. 5; 490-501. 
9) Leers-Sucheta, S., Morohashi, K., Mason, J. I. and Melner, M. H (1997) Synergistic activation of the human type II 3beta-hydroxysteroid dehydrogenase/delta5-delta4 isomerase promoter by the transcription factor steroidogenic factor-1/adrenal 4-binding protein and phorbol ester. J. Biol. Chem. 272; 7960-7967.

10) Mellon, S. H., Bair, S. R. and Monis, H. (1995) P450c11B3 mRNA, transcribed from a third $\mathrm{P} 450 \mathrm{c} 11$ gene, is expressed in a tissue-specific, developmentally, and hormonally regulated fashion in the rodent adrenal and encodes a protein with both 11 hydroxylase and 18-hydroxylase activities. J. Biol. Chem. 270; 1643-1649.

11) Mitani, F., Mukai, K., Miyamoto, H., Suematsu, M. and Ishimura, Y. (1999) Development of functional zonation in the rat adrenal cortex. Endocrinology 140; 3342-3353.

12) Morohashi, K., Zanger, U. M., Honda, S., Hara, M., Waterman, M. R. and Omura, T. (1993) Activation of CYP11A and CYP11B gene promoters by the steroidogenic cell-specific transcription factor, Ad4BP. Mol. Endocrinol. 7; 1196-1204.

13) Morohashi, K., Iida, H., Nomura, M., Hatano, O., Honda, S., Tsukiyama, T., Niwa, O., Hara, T., Takakusu, A., Shibata, Y. and Omura, T. (1994) Functional difference between Ad4BP and ELP, and their distributions in steroidogenic tissues. Mol. Endocrinol. 8; 643-653.

14) Murakoshi, M., Osamura, R. Y. and Watanabe, K. (2000) Immunolocalization of glutathione-peroxidase (GSH-PO) in the steroid-producing organs and prostate. Acta Histochem. Cytochem. $33 ; 405-412$.

15) Nachtigal, M. W., Hirokawa, Y., Enyeart-VanHouten, D. L., Flanagan, J. N., Hammer, G. D. and Ingraham, H. A. (1998) Wilms' tumor 1 and Dax-1 modulate the orphan nuclear receptor SF-1 in sex-specific gene expression. Cell 93; 445-454.

16) Narasaka, T., Suzuki, T., Moriya, T. and Sasano, H. (2001) Temporal and spatial distribution of corticosteroidogenic enzymes immunoreactivity in developing human adrenal. Mol. Cell. Endocrinol. 174; 111-120.

17) Orth, D. N., Kovacs, W. J. and Rowan, D. C. (1998) The adrenal cortex. In "Willeam's Textbook of Endocrinology", 9th ed., ed. by J. D. Wilson and D. W. Foster, Saunders, Philadelphia, pp. 489-619.

18) Pankratz, D. S. (1931) The development of the suprarenal gland in the albino rat, with consideration of its possible relation to the origin of movements. Anat. Rec. 49; 31-49.

19) Parker, K. L. and Schimmer, B. P. (1997) Steroidogenic factor 1: a key determinant of endocrine development and function. Endocr. Rev. 18; 361-377.

20) Rice, D. A., Kirkman, M. S., Aitken, L. D., Mouw, A. R., Schimmer, B. P. and Parker, K. L. (1990) Analysis of the promoter region of the gene encoding mouse cholesterol side-chain cleavage enzyme. J. Biol. Chem. 265; 11713-11720.

21) Scazzocchio, C. (2000) The fungal GATA factors. Curr. Opin Microbiol. 3; 126-131.

22) Simon, M. C. (1995) Gotta have GATA. Nat. Genet. 11; 9-11.

23) Sugawara, T., Holt, J. A., Kiriakidou, M. and Strauss, J. F. 3rd (1996) Steroidogenic factor 1-dependent promoter activity of the human steroidogenic acute regulatory protein (StAR) gene. Biochemistry 35; 9052-9059.

24) Swain, A. and Lovell-Badge, R. (1999) Mammalian sex determination: a molecular drama. Genes Dev. 13; 755-767.

25) Takayama, K., Morohashi, K., Honda, S., Hara, N. and Omura, T. (1994) Contribution of Ad4BP, a steroidogenic cell-specific transcription factor, to regulation of the human CYP11A and bovine CYP11B genes through their distal promoters. J. Biochem. (Tokyo) 116; 193-203.

26) Tremblay, J. J. and Viger, R. S. (2001) GATA factors differentially activate multiple gonadal promoters through conserved GATA regulatory elements. Endocrinology 142; 977-986.

27) Weiss, M. J. and Orkin, S. H. (1995) GATA transcription factors: key regulators of hematopoiesis. Exp. Hematol. 23; 99-107.

28) Zhang, P. and Mellon, S. H. (1996) The orphan nuclear receptor steroidogenic factor-1 regulates the cyclic adenosine 3',5'-monophosphate-mediated transcriptional activation of rat cytochrome P450c17 (17 alpha-hydroxylase/c17-20 lyase). Mol. Endocrinol. $10 ; 147-158$. 